\title{
Detailed Abundances for Field Stars Surrounding the LMC Cluster Hodge 11
}

\author{
Renee Mateluna ${ }^{1}$, Douglas Geisler and Sandro Villanova \\ Departamento de Astronomia \\ Universidad de Concepción \\ Esteban Iturra s/n Barrio Universitario, Concepción, Chile \\ ${ }^{1}$ email: renee@astro-udec.cl
}

\begin{abstract}
We present results of a detailed model atmosphere abundance analysis for a variety of elements, including $\mathrm{Fe}, \mathrm{Ca}, \mathrm{Si}, \mathrm{Ti}, \mathrm{Sc}, \mathrm{Ni}, \mathrm{Cr}$ and $\mathrm{Ba}$ for a number of giants in the surrounding field of the LMC cluster H11, obtained from high resolution FLAMES@VLT spectra.
\end{abstract}

Keywords. abundances, Hodge11, Large Magellanic Cloud

\section{Introduction}

The Large Magellanic Cloud (LMC) exhibits an array of star formation processes and episodes. This galaxy provides an excellent opportunity to study the formation and chemical enrichment history of similar galaxies in great detail. Additionally, the Lambda CDM paradigm of galaxy formation suggests that galaxies like the Milky Way form their halos through the accretion/merger of numerous dwarf satellites. Therefore understanding the formation history and chemical evolution of the LMC is an important step in understanding these merger events and their role in Galaxy formation. One of the most direct ways to learn about a galaxy's chemical evolution and star formation history is through the study of its clusters. Both their ages and abundances are relatively easily determined, however for the LMC a huge gap in the cluster age metallicity relation is present (DaCosta (1991), (2002) and Geisler et al. (1997)) with only one known cluster in it. If we want to investigate the chemical evolution during this period, we must use field stars.

\section{Data and Results}

The data was obtained with the high resolution spectrograph FLAMES (GIRAFFE: R 25000 and UVES: R 47000) at VLT. The abundances of Ca and Ti (alpha-elements) for LMC field stars are presented in Fig. 1. The abundances for $\mathrm{Ni}$ and $\mathrm{Cr}$ (iron-peak elements) are presented in Fig. 3. The abundance for Sc are presented in Fig. 2. All these results are presented in comparison with Pompeia et al. 2008 data for the general LMC field. The abundances for all these elements were obtained with an LTE model atmosphere analysis.

\section{Conclusions and Future Work}

The obtained abundances so far are in good agreement with the results of Pompeia et al. 2008 for the LMC field stars (Fig. 1, 2 and 3). The next step is to obtain high resolution spectroscopy of a sub-sample of clusters and field stars. We already have data for four of them (NGC2257, Hodge 11, NGC 1841, NGC 1718) with GIRAFFE and UVES 

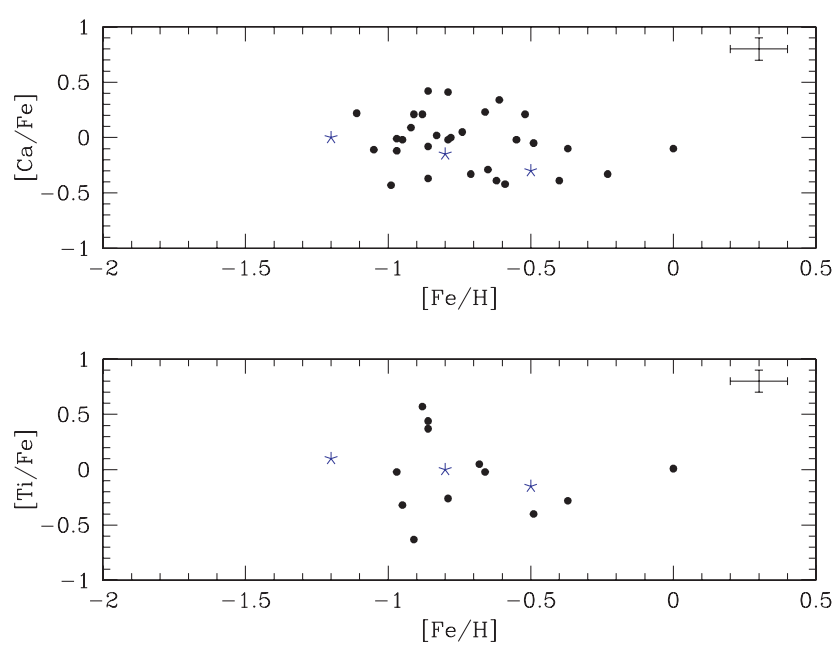

Figure 1. Abundances of Ca and Ti compared to Fe for LMC field stars. Black dots: our data, the crosses are the mean values for LMC from Pompeia et al. 2008.
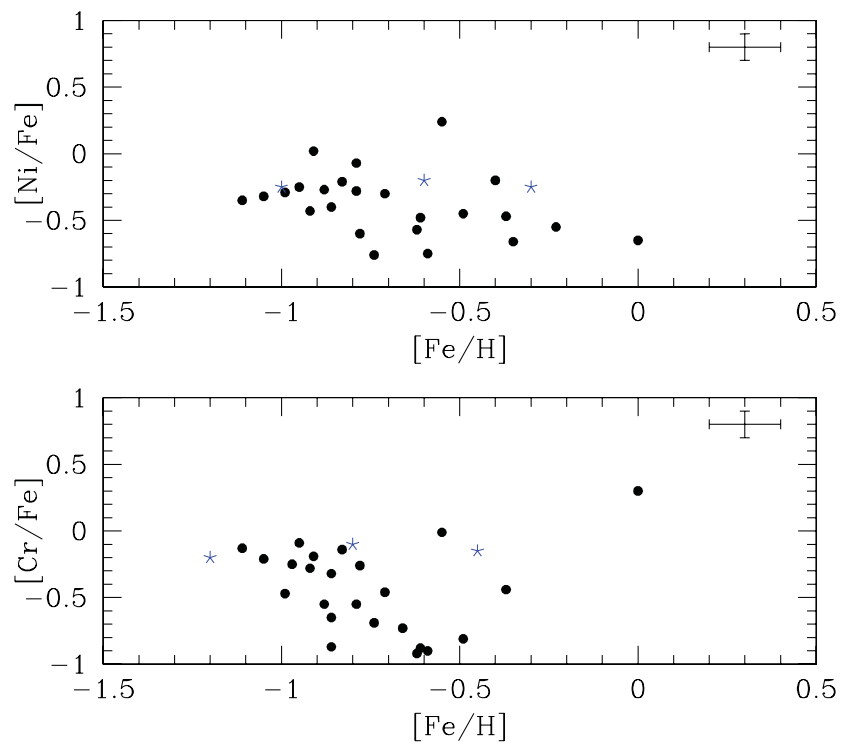

Figure 2. Abundances of iron-peak elements (Ni and $\mathrm{Cr}$ ) compared to Fe for LMC field stars. Black dots:are the mean values for LMC from Pompeia et al. 2008.

(FLAMES@VLT). Spectroscopic data will be coupled with deep wide-field photometry obtained with MOSAIC at CTIO $4 \mathrm{~m}$, in order to have an homogeneous and complete database and derive atmospheric parameters. These clusters were selected because they span the full range of age $(2-13 \mathrm{Gyr})$ and metallicities $(-0.4$ to -2.0$)$ of the FORS2 sample (Grocholski et al. 2006). Our goal is to derive abundances for many elements, including $\mathrm{Na}$, alpha ( $\mathrm{Si}, \mathrm{Ca}, \mathrm{Ti}$ ), iron-peak (V, Cr, Fe, Ni, Cu), and sprocess ( $\mathrm{Y}$ and $\mathrm{Ba}$ ). The detailed age/abundance relations for these elements, along with information about the relative contribution from AGB stars and Type I and II SNe, will be used to help constrain the chemical evolution of the LMC. 


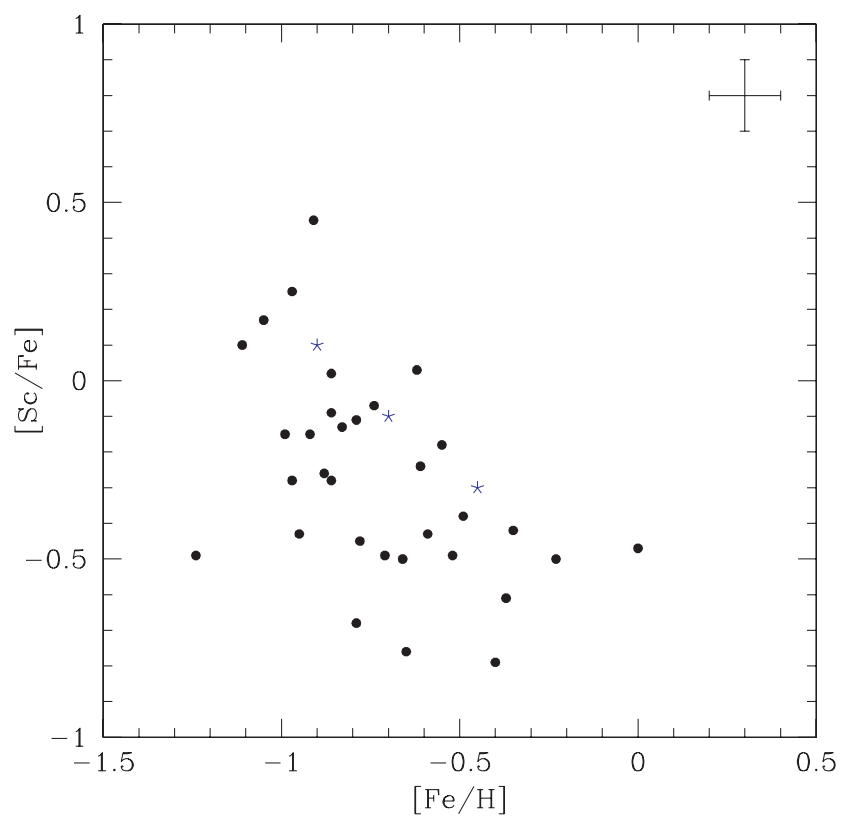

Figure 3. Abundances of Sc compared to Fe for LMC field stars. Black dots: our data, the crosses are the mean values for LMC from Pompeia et al. (2008).

\section{Acknowledgement}

Thanks to Conicyt for my PhD Scholarship. To SOCHIAS, to ALMA-Conicyt fund (Project n 31070007), to Universidad de Concepcin, to MECESUP fund and to CATABASAL for their financial support in this meeting.

\section{References}

Da Costa, G. S. 1991, IAU Symp, 148, 183

Da Costa, G. S. 2002, IAU Symp, 207, 83

Geisler, D., Bica, E., Dottori, H., Clairá, J. J., Piatti, A. E., \& Santos, J. F. C., Jr. 1997, AJ, 114, 1920

Grocholski, A. J., Cole, A. A., Sarajedini, A., Geisler, D., \& Smith, V. V. 2006, AJ, 132, 1630

Pompeia, L., Hill, V., Spite, M., Cole, A., Primas, F., Romanicello, M., Pasquini, L., Cioni, M.-R., \& Smecker Hane, T. 2008, A\&BA, 480, 379 